\title{
Increased Adipsin is Associated with Carotid Intima Media Thickness and Metabolic Disturbances in Polycystic Ovary Syndrome
}

. Gokcen UNAL KOCABAS ${ }^{1}$, Ozlem GURSOY CALAN ${ }^{2}$, Mehmet CALAN ${ }^{1}$, Pinar YESIL SENSES ${ }^{3}$, Ebru OZDEN ${ }^{4}$; Kerime Rumeysa SARI ${ }^{4}$, Merve KOCAR ${ }^{4}$, Cetin IMAMOGLU5, Yasar Mehmet SENSES ${ }^{6}$, Giray BOZKAYA ${ }^{7}$, Oktay BILGIR ${ }^{4}$

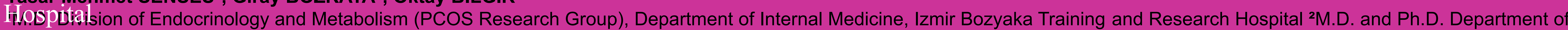

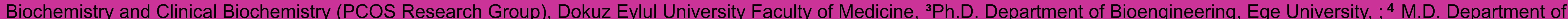
Internal Medicine (PCOS Research Group), Izmir Bozyaka Training and Research Hospital, ${ }^{5} \mathrm{M}$.D. Department of Radiology, Izmir Bozyaka Training and Research Hospital; ${ }^{6}$ M.Sc. Department of Biotechnology, Ege University; ${ }^{7}$ M.D. Department of Biochemistry and Clinical Biochemistry, Izmir Bozyaka Training and Research Hospital. Author

\section{Objectives:}

Adipsin, a protein secreted mainly from the adipose tissue, is a structural homologous of complement factor $D$, a rate-limiting enzyme of the alternative complement system.

Growing evidence suggests that the alternative complement system plays a role both in the regulation of energy homeostasis and the atherosclerotic process. Polycystic ovary syndrome (PCOS) is an inflammatory based-metabolic disease. The main objective of this study is to ascertain whether circulating adipsin levels are altered in women with PCOS, and whether there is association between adipsin and metabolic parameters or carotid intima media thickness (CIMT).

\section{Methods:}

Participants: 144 women with PCOS and 144 age- and BMImatched controls without PCOS were recruited for this crosssectional study.

Main Outcome Measures: Circulating adipsin levels were measured using ELISA. Metabolic, hormonal parameters and CIMT were also determined.

Circulating adipsin levels were significantly elevated in women with PCOS compared with controls (91.52 \pm 14.11 vs. $60.31 \pm 9.71 \mathrm{ng} / \mathrm{ml}, P<0.001)$. Adipsin levels positively correlated with BMI, homeostasis model assessment of insulin resistance (HOMA-IR), total \& free-

testosterone, high sensitivity C-reactive protein (hs-CRP), triglycerides and CIMT. Multivariate logistic regression analyses revealed that the odds ratio for PCOS was 3.25 for patients in the highest quartile of adipsin compared with those in the lowest quartile (OR=3.25, 95\% Cl=2.64-4.00, $P=0.016)$. Our findings further indicate that BMI, HOMA-IR, hs-CRP and free-testosterone are independent factors influencing serum adipsin levels and that adipsin is an independent predictor for CIMT..

\section{Conclusions:}

Adipsin might contribute to the development PCOS as well as the cardiovascular risks and metabolic disturbances associated with the disease. 